\title{
A Randomized Controlled Pilot Study to Evaluate a Technology Platform for the Assisted Living of People with Dementia and their Carers
}

\author{
Mariam Torkamani $^{\mathrm{a}, *}$, Louise McDonald ${ }^{\mathrm{a}}$, Ignasi Saez Aguayo ${ }^{\mathrm{b}}$, Christos Kanios ${ }^{\mathrm{c}}$, \\ Maria-Nefeli Katsanou ${ }^{c}$, Laura Madeley ${ }^{\mathrm{d}}$, Patricia D. Limousin ${ }^{\mathrm{a}}$, Andrew J. Lees ${ }^{\mathrm{e}}$, Maria Haritou ${ }^{\mathrm{f}}$, \\ Marjan Jahanshahi ${ }^{\mathrm{a}}$ and the ALADDIN Collaborative Group ${ }^{1}$ \\ ${ }^{a}$ Sobell Department of Motor Neuroscience \& Movement Disorders, UCL Institute of Neurology \& The National \\ Hospital for Neurology and Neurosurgery, London, UK \\ ${ }^{\mathrm{b}}$ Badalona Serveis Assistencials, Badalona, Spain \\ ${ }^{\mathrm{c}}$ The Psychiatric Hospital of Attica, Athens, Greece \\ ${ }^{\mathrm{d}}$ Newham University Hospital, London, UK \\ ${ }^{\mathrm{e}}$ Reta Lila Weston Institute of Neurological Studies, UCL Institute of Neurology, London, UK \\ ${ }^{\mathrm{f}}$ Institute of Communications \& Computer Systems, Athens, Greece
}

Accepted 2 February 2014

\begin{abstract}
The use of telemedicine is becoming increasingly popular in assisting with the home management of People with Dementia ( $\mathrm{PwD}$ ) by offering services to the carers that may enhance their ability to care for their relative for longer. A computerized platform, ALADDIN, was evaluated in its usefulness to reduce carer burden and distress and to improve their quality of life, in an attempt to delay institutionalization of PwD. ALADDIN offers educational material about dementia to carers and provides the opportunity to contact other carers and clinicians. ALADDIN also facilitates remote monitoring of the PwD and their carers by the clinicians to enable speedy delivery of appropriate intervention. The ALADDIN platform was piloted at three European sites, and used by thirty carers of PwD living in the community (platform group). The platform group and a control group of thirty $\mathrm{PwD}$ and their carers were assessed at baseline, 3 months, and 6 months. The results showed a significant improvement in the quality of life of the carers in the platform group, with some reduction in carer burden and distress. The platform was useful in monitoring the patients and facilitating contact with other professionals. Access to and use of the ALADDIN platform was rated positively by carers and clinicians. The ALADDIN platform's usefulness and applicability for prolonging the home management of PwD are discussed.
\end{abstract}

Keywords: Carer burden, dementia, distress, institutionalization, quality of life, telecare, telemedicine

\footnotetext{
*Correspondence to: Mariam Torkamani, Sobell Department of Motor Neuroscience \& Movement Disorders, UCL Institute of Neurology \& The National Hospital for Neurology and Neurosurgery, Queen Square, London WC1N 3BG, UK. Tel.: +44 0203448 8733; Fax: +44 0207419 1860; E-mail: m.torkamani@ucl.ac.uk.

${ }^{1}$ The ALLADIN Collaborative Group included: Raffaella Calati, Yiannis Christopoulos, Francesco D'Andria, Gianpaolo Perego, Vassiliki Psarra, Alessandro Serretti, and Marios Tigas.
}

\section{INTRODUCTION}

\section{Background}

The growing prevalence of dementia as a result of the increase in the aging population [1] has led to concerns regarding the delivery of care and the rising costs of it. At present around $60 \%$ of people with dementia 
(PwD) live in the community and are looked after by informal (family) carers [2] and the care of the other $40 \%$ that are institutionalized cost the economy around \$315billion per annum worldwide [3]. Although the care of PwD at home is beneficial for the PwD and the wider society, it places a great burden on the informal carer. The informal carers of PwD typically experience high risks of depression [4-6], early mortality [7], and social isolation [8], as well as reduced quality of life (QoL) [9] and employment [8]. As a result, the carer's burden, distress, and QoL are some of the main issues that need to be addressed in the care of PwD at home.

In the face of greater demands on health services, there is now growing interest in the use of telemedicine systems for management of dementia at home by offering a range of services that can be used to support carers of PwD. Many studies report on the usefulness of telemedicine interventions for carers of PwD, including: non-internet computer based forums [10], computer automated telephone support systems [11], computerized systems for accessing information [12], internet library [13], and internet-based videoconferencing [14]. While the outcomes of these studies are inconsistent and show that the interventions benefit some but not all carers, they highlight the potential of telemedicine interventions.

The current study is a multi-center randomized controlled evaluation of a technology platform specifically designed for PwD living at home and their carers. The system is entitled: 'A technology pLatform for the Assisted living of Dementia elDerly INdividuals and their carers' (ALADDIN).

\section{ALADDIN}

ALADDIN is a computerized platform designed to offer avenues of support and information to the carer. It also manages and communicates information related to the PwD and their carers from their home to the clinicians, facilitating distant monitoring. ALADDIN has four key features: 'ALADDIN TV', 'SOCIAL NETWORKING', 'MY TASKS', and 'CONTACT US'.

1) 'ALADDIN TV' provides information and educational material about dementia, as well as musical entertainment and relaxation and exercise techniques. The provision of information about dementia online has been shown to be beneficial for carers [15].

2) The 'SOCIAL NETWORKING' feature provides a forum for carers using ALADDIN to communicate with each other. Contact with other carers is recommended by NICE guidelines [16].
3) 'MY TASKS' is the distant monitoring feature of ALADDIN, where carers complete questionnaires about their own and their relatives' health. Their responses can subsequently generate clinical alerts based on set parameters, comparing new responses to previous data, resulting in the immediate detection of change by the clinicians monitoring the system. This facilitates the speedy delivery of appropriate interventions to reduce risks of emergencies and/or institutionalization in the future.

4) The 'CONTACT US' feature allows the carer to alert the clinical site and/or generate a request for contact. Such frequent and improved communication between the carer and health professionals enhances the carer's ability to better manage PwD's problematic behaviors [17].

Through the distant monitoring of the carers and the PwD (MY TASKS), provision of pertinent information about dementia and caring, and access to relaxation and exercise routines (ALADDIN TV) and avenues of support ( 'CONTACT US' and 'SOCIAL NETWORKING' features), ALADDIN aims to reduce carer burden and distress and enhance their QoL. Thus the present study is a randomized controlled evaluation of the ALADDIN system for continued care of PwD at home.

\section{METHODS}

\section{Design}

A multi-center randomized controlled pilot study was undertaken at three European sites: The National Hospital for Neurology and Neurosurgery (NHNN), UK; Badalona Serveis Assistencials (BSA), Spain; and the Psychiatric Hospital of Attica (PHA), Greece. The study was approved by National Research Ethics Service Committee, UK.

\section{Participants}

Hospital outpatients identified as having dementia were screened for functional dependency and cognitive impairment using the Barthel Index (BI) [18] measure of disability and the Mini-Mental State Examination (MMSE) [19] measure of cognition. Patients living at home with a full time carer, a BI score of at least 35 (indicating some degree of independence), and a MMSE score of at least 9 and no more than 21 (indicating moderate to mild cognitive impairment) were recruited. Patients either had dementia as their 
Table 1

Mean and (standard deviation) of demographic and clinical details of patients in the Platform and Control groups, and $\mathrm{p}$ value for the independent $t$-test comparisons

\begin{tabular}{lcccc}
\hline & $\begin{array}{c}\text { Platform } \\
(n=30)\end{array}$ & $\begin{array}{c}\text { Control } \\
(n=30)\end{array}$ & $\begin{array}{c}\text { All } \\
(N=60)\end{array}$ & $p$ \\
\hline Carer & & & & \\
Age & $57.57(12.50)$ & $63.93(14.74)$ & $60.69(13.90)$ & 0.079 \\
Patient & & & & \\
Age & $78.60(7.49)$ & $77.47(6.36)$ & $78.03(6.91)$ & 0.530 \\
Disease & $3.7(2.48)$ & $2.7(1.73)$ & $3.2(2.18)$ & 0.075 \\
$\quad$ duration & & & & \\
BI score & $63.5(22.94)$ & $73.83(26.35)$ & $68.67(25.04)$ & 0.111 \\
MMSE score & $18.9(4.55)$ & $19.73(5.46)$ & $19.32(5.00)$ & 0.523 \\
Gender & $45 \%$ male & $55 \%$ male & $55 \%$ male & 0.201 \\
$\quad$ variation & $55 \%$ female & $45 \%$ female & $45 \%$ female & \\
\hline
\end{tabular}

BI, Barthel Index; MMSE, Mini-Mental State Examination.

primary condition or dementia as part of Parkinson's disease. Informed consent was sought from all participants (patients and their carers), and an assent form was signed by the carer if the PwD lacked capacity to provide consent. The general practitioners of all patients were informed of their participation in the study. Twenty participants (patients and their carers) were recruited at each site. Ten participants from each site were then randomly allocated to the control group $(n=30)$, and ten to the platform group $(n=30)$. There were no significant differences in key demographic variables between the two groups at the time of recruitment (See Table 1).

\section{Procedure}

At each pilot site, one clinician and one technician were involved in the project. The project ran for 6 months, and all participants were assessed in their homes at three time points: baseline, 3 months, and 6 months. The platform participants were visited prior to the baseline assessment and provided with access to the ALADDIN platform to use for the duration of the study. Internet connection and laptops were provided where necessary. The carers were trained so that they could navigate the system and complete 'MY TASKS' monitoring of the PwD and their own mental state and burden. Data from 'MY TASKS' were used solely for the purposes of identifying change during the project period, and were not used as part of the evaluation of change in patients or their carers. Being the primary users of ALADDIN, the carers chose the schedule of their tasks. The system was monitored twice daily by the clinical teams. The participants in the control group were only assessed at the three time points, without any further contact or intervention.

\section{Measuring instruments}

The assessments conducted at baseline, 3 months, and 6 months on both groups were identical. The assessments completed with the carers were used for evaluating the usefulness of ALADDIN to reduce carer burden and distress and enhance QoL. The assessments of PwD were used solely for the description of sample which may have influenced carer outcomes.

\section{Assessments completed with carer}

Zarit Burden Interview (Zarit) [20]: a 22 item instrument which measures carer's perceived burden of providing care. With a total range of $0-88$, higher scores indicate greater burden.

Neuropsychiatric Inventory (NPI) [21]: this interview schedule with carers is used to measure occurrence of psychiatric and behavioral problems in the PwD. It assesses 12 domains: hallucinations, delusions, agitation/aggression, dysphoria/depression, anxiety, irritability, disinhibition, euphoria, apathy, aberrant motor behaviors, sleep, and appetite. The frequency and severity of the behavior in question are multiplied and added to give the total NPI value, and the carer's distress scores are added to give a separate sum.

Beck Depression Inventory (BDI) [22]: a 21 item scale, measuring somatic, cognitive, affective and behavioral aspects of depression. With a possible range of $0-63$, scores of $0-9,10-18,19-25$, and 30+ represent normal, mild, moderate, and severe depression respectively.

Zung Depression Self Rating Scale (Zung) [23]: a 20 item questionnaire covering affective, psychological, and somatic symptoms of depression. Score range is $20-80$, with higher scores representing more severe depression. A score above 50 indicates clinical depression outside the normal range.

EuroQoL (EQ5D) [24]: a 5 item questionnaire covering five dimensions of QoL: mobility, self-care, usual activities, pain/discomfort, and anxiety/depression. The sub-scores can be combined to give a summary index value of $0-1$. The EQ5D also includes a visual analogue scale for rating 'current health state'. Lower scores represent poorer QoL for both.

Quality of Life Scale (QOLS) [25]: a 16 item questionnaire measuring six domains of QoL: material and physical well-being, relationships with others, social, community, and civic activities, personal development and fulfilment, and recreation. With a range of 16-112, higher scores indicate better QoL.

Due to the European collaborative nature of this project, and the fact that some scales were not available or validated in some languages, QoL and mood 
were assessed using different scales. To measure QoL, QOLS was used by two of the three clinical sites and EQ5D was used by one. Similarly, to measure carer's mood, BDI was used by one site at each assessment, and Zung was used by two sites at the final assessment only. Consequently, carers QoL are reported using both the EQ5D and QOLS, and the cut off points for the depression scales (Normal, Mild, Moderate, Severe) were used to make the BDI and Zung depression scales comparable.

\section{Assessments completed with PwD}

The patient's blood pressure and weight were recorded on the day of the assessment, and the PwD were tested on a number of assessment tools including the following:

Mini-Mental State Examination (MMSE) [19]: a 30 item measure of global cognitive functioning covering: orientation, memory, language, and executive function. The score range is $0-30$, with higher scores representing better cognitive functioning. Scores below 24 are considered to indicate cognitive impairment.

Mattis Dementia Rating Scale 2 (DRS2) [26]: a 36 item test of cognitive function covering: attention, initiation-perseveration, construction, conceptualization, and memory. The score range is $0-144$, where higher scores represent better performance.

Blessed Dementia Rating Scale (BDRS) [27]: a 22 item questionnaire covering: everyday activities, self care habits, and changes in personality, interests, and drives. Each item is rated from 0 (total capacity) to 0.5 (intermittent incapacity) to 1 (total incapacity).

Clinical Dementia Rating Scale (CDRS) [28]: a 6 item questionnaire measuring: memory, orientation, judgment and problem solving, community affairs, home and hobbies, and personal care. Each item is scored on a scale of 0 (no impairment) to 3 (severe impairment). Memory is the main category, and provided that at least two of the other items have the same severity score as the memory subtest, that score is considered to represent the overall CDRS score; otherwise the score given to the majority of the items becomes the overall CDRS score.

Revised Memory and Behavior Problems Checklist $(M B P C)$ [29]: a 24 item questionnaire covering: memory related problems, affective distress and disruptive behaviors, and their impact on the carer. With a total range of 0-96, the higher the score, the more frequent the problem. The carer's distress can also be calculated in the same way.

Geriatric Depression Scale (GDS) [30]: a 15 item measure of depression. Each answer that is indicative of depression is given a score of 1 . With a total range of $0-15$, scores above 5 indicate depression.

Barthel Index (BI) [18]: a measure of functional disability looking at independence in activities of daily living. The items cover: feeding, bathing, grooming, dressing, bowel control, bladder control, toileting, chair transfer, ambulation, and stair climbing. The ten items give a total range of $0-100$, with higher scores representing increasing dependence.

Lawton Activities of Daily Living (LADL) [31]: a measure of independent living covering functioning in 8 domains: ability to use telephone, laundry, shopping, mode of transportation, food preparation, responsibility for own medication, housekeeping, and ability to handle finances. The eight items give a total range of $0-8$, with higher scores showing increasing independence.

Charlson's Comorbidity Index (CCI) [32]: a 19 item index, consisting of a range of morbid conditions. Each condition has a pre-assigned score depending on risk of dying associated with it. All scores are added together to give the predicted value of mortality for the patient. The higher the score, the higher the predicted mortality.

\section{Evaluation of the ALADDIN platform}

In addition to the assessment of carers, to evaluate the ALADDIN platform's usefulness, two specific questionnaires looking at the experiences of its main users were developed. The main users of the platform were the carers who completed the tasks and navigated the system, and the clinicians who set the tasks and monitored the system. The carer and clinician 'satisfaction questionnaires' consisted of rating scales measuring the ease of use of the system, the user's willingness to continue using it, and recommending it to a friend (carer) or substituting it with in-person consultations (clinician). The questionnaires also assessed the carer's perception of ALADDIN in enhancing/increasing awareness of their own and their relative's wellbeing, and other questions related to the frequency of the tasks and their toll on increasing duties. The clinicians were asked about their thoughts on the automatically generated alerts, the overall value of ALADDIN and its suitability and potential for the future management of PwD in their services.

\section{Statistical analyses}

A series of independent $t$-tests and analysis of covariance (ANCOVA) were conducted using the SPSS software for Windows (version 20). To evaluate 


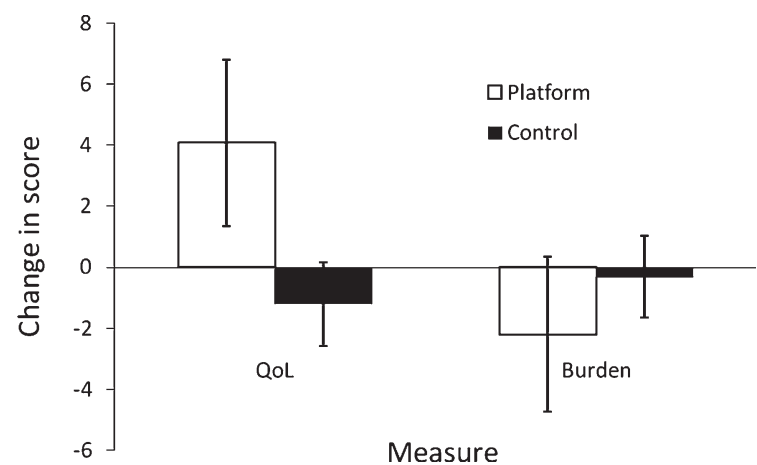

Fig. 1. Changes in the quality of life $(\mathrm{QoL})$ and burden measures for the carers in the Platform and Control groups over the six month period of study. The mean and standard error of the mean (error bars).

changes across the two groups over time, a repeated measures Mixed ANCOVA was conducted with Group (Platform versus Control) and Time of Assessment (3 and 6 months) as the between and within subjects variables respectively. Scores from the baseline assessment were used as the covariates.

\section{RESULTS}

Carer burden, distress, and quality of life

A series of independent $t$-tests were carried out to identify any differences between the carers in the platform and control groups at baseline. The comparisons revealed significant differences in carer burden [Zarit; $t(58)=2.063, p=0.044]$ and QoL [QOLS, $t(58)=-2.286, p=0.026]$ (see Table 2), showing higher burden and worse QoL for carers in the platform group than those in the control group. These results were used as covariates in subsequent analysis of variance.

A number of two-way ANCOVAs were conducted to look at the effect of Group and Time of Assessment, and any interaction between the two variables, on carer burden, distress, and QoL.

Although there were no significant differences in ratings of carer burden between the two groups $(p>0.05$ for all), to determine the success of ALADDIN in reducing carer burden, the average change in the relevant measure was calculated (See Fig. 1). This showed that during the course of the pilot study, on average, the burden of caring was reduced more in carers in the platform group $(M=-2.2 ; S D=13.01)$ than those in the control group $(M=-0.3 ; S D=.27)$.

The results showed no significant main effects or interactions on the carer's distress associated with their

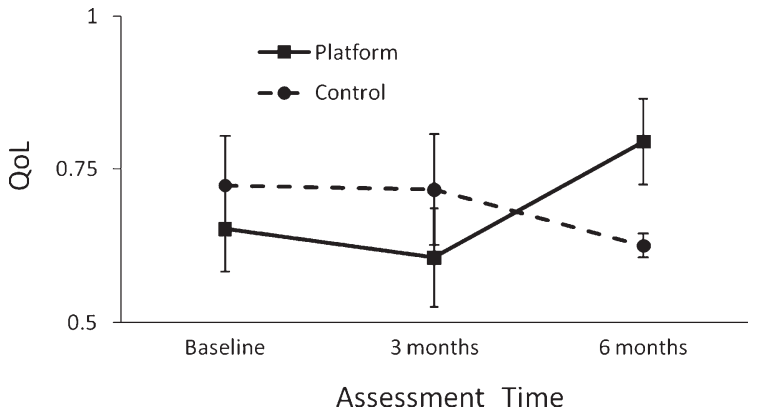

Fig. 2. Changes in the quality of life (QoL) of carers in the Platform and Control groups as measured by EQ5D over the six month period of study. The mean and standard error of the mean (error bars).

relatives' neuropsychiatric symptoms (NPI carer distress ratings) (all $p>0.05$ ).

ANOVA was not possible for depression scores for all participants due to missing data. Thus we compared the severity of depression at the final assessment, and although the group differences did not reach statistical significance $\left[\chi^{2}(2,57)=1.33, p=0.514\right]$, there were some notable differences. While $75 \%$ of the carers in the platform group scored in the normal range and $25 \%$ had mild depression, $66 \%$ of carers in the control group scored in the normal range, $31 \%$ had mild depression, and one had moderate depression (3\%).

Furthermore, despite reports of poorer QoL in carers in the platform group at baseline relative to controls, a significant Group $x$ Time of Assessment interaction was found for the EQ5D measure of QoL $[F(1,16)=5.9317, p=0.027)]$. This significant interaction was due to the improvement in the QoL of carers in the platform group at 6 months (see Fig. 2). Since QoL was measured using QOLS by two of the clinical sites and EQ5D by one site only, the scores were converted into percentages to enable comparisons. The average change in carers' QoL from baseline to six months was a $4.1 \%$ gain $(S D=14.24)$ for carers in the platform group, and a $1.2 \%$ loss $(S D=-7.53)$ for carers in the control group (see Fig. 1)

To investigate whether the improvements reported above were related to the platform carers' use of ALADDIN, we looked at their responses from the carer-satisfaction questionnaire. A large percentage of the carers felt that ALADDIN: gave them more confidence because of the frequent monitoring of their relative $(64 \%)$; made them more aware of their relative's health $(53 \%)$; and provided them with information about dementia (56\%). Also while some carers felt that ALADDIN reduced their anxiety about their relative's health $(46 \%)$, most considered ALADDIN as having the potential to improve their QoL $(60 \%)$. 


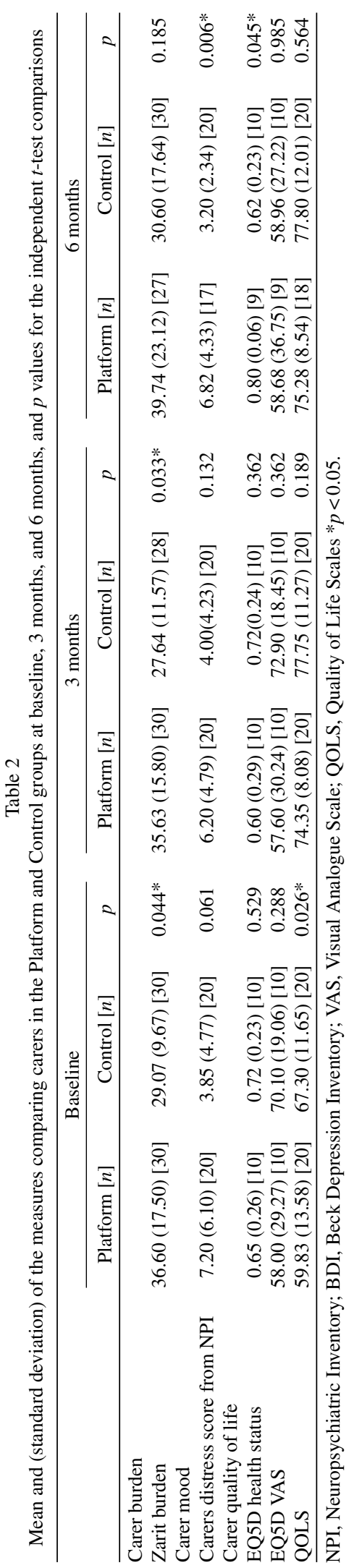




\section{Change in PwD}

A number of independent $t$-tests were completed to identify any significant differences at baseline between PwD in the two groups. The analyses revealed significant differences on the patients' dementia severity [CDRS; $t(58)=1.837, p=0.051]$ and neuropsychiatric symptoms [NPI; $t(57)=2.129, p=0.038]$, showing higher severity in patients randomly allocated to the platform group. These group differences remained significant at 3 and 6 months (all $p<0.05$ ).

In addition, a series of two-way ANCOVAs were conducted to identify any changes between PwD in the platform and control groups over time. There were no significant interactions, however the results showed significant main effects for Group on the MMSE $[F(1,52)=7.189, p=0.010]$ and $\operatorname{CDR}[F(1,54)=4.600, p=0.036]$ scores, and for the patient's weight $[F(1,34)=7.4470, p=0.010]$. Inspection of the means indicate poorer outcomes in patients in the platform group on the MMSE and CDR (see Fig. 3a, b) scores. On the other hand, however, the patients in the platform group showed a weight gain (see Fig. 3c). No other significant interactions or main effects were observed.

\section{DISCUSSION}

The aim of this study was to investigate whether access to information and support through a telemedicine system, and frequent monitoring of PwD and their carer, could help reduce carer burden and distress and improve the carer's QoL. In comparison to a control group, the results showed some reduction in carer burden and distress and improvement in the QoL of carers that were given access to the computerized platform ALADDIN.

Despite the random allocation, the participants in the platform group showed poorer outcomes at baseline relative to the controls. However a key theme emerging from our findings is that despite the variance at baseline, during the course of the study period or at the final assessment, the participants in the platform group showed greater improvements relative to the controls.

In line with previous studies looking at the effectiveness of telemedicine interventions in reducing carer burden [12-14], we did not find any statistically significant results for carer burden. However we did observe a greater reduction of burden during the course of the study in carers using ALADDIN relative to the controls, and the carers' overall response to using
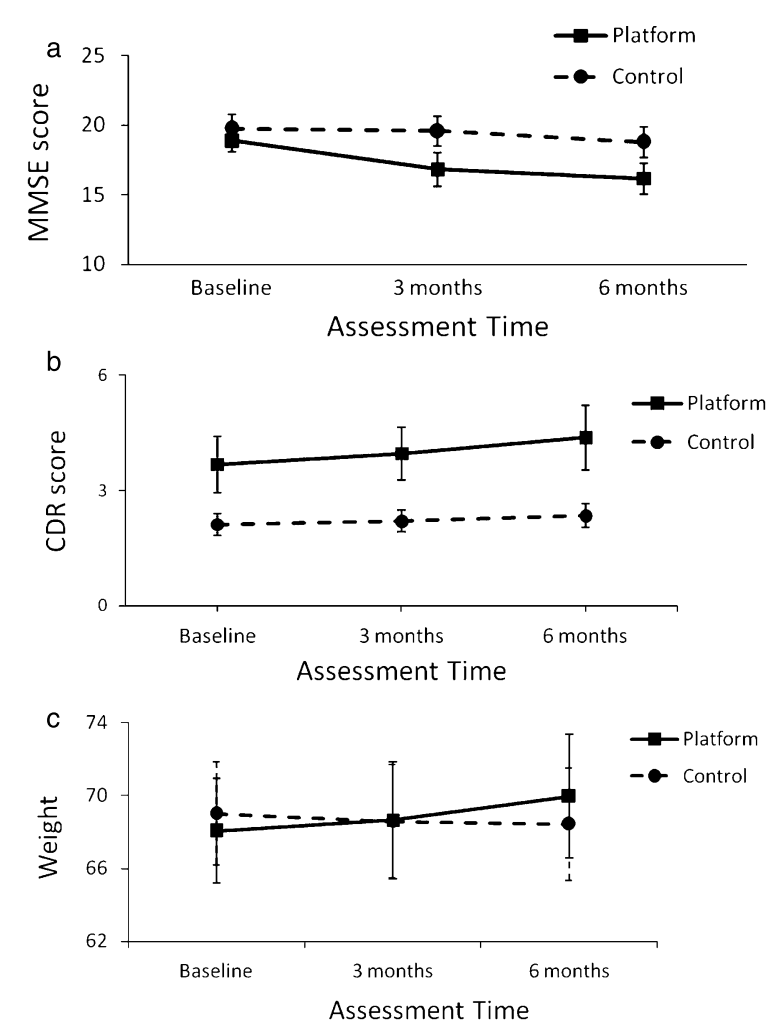

Fig. 3. Changes in the average scores of the patients in the Platform and Control groups on the MMSE (a) and CDR (b), and their weight (c) over the six month period of the study. The mean and standard error of the mean (Error bars).

ALADDIN was also positive. Thus, continued use of the system may have introduced significant results.

Furthermore, contrary to previous studies which have shown improvements in self-efficacy and confidence [13], but not depression [11, 12] in carers using telemedicine interventions, in our sample the carers in the platform group showed slightly milder forms of depression than those in the control group at the final assessment. Although due to missing data we were unable to determine if this difference existed at baseline, feedback from the carers using ALADDIN suggested that the provision of information and the frequent monitoring of their relative with dementia through ALADDIN reduced their anxieties and gave them more confidence.

In addition, our results showed a significant interaction on carer QoL. Inspection of the means indicate that despite lower QoL at baseline in the platform group, 6 months after the use of ALADDIN they had higher self-reported QoL than those in the control group. Figure 2 illustrates a non-parallel pattern in these scores, indicating that although there is a small 
drop in QoL for both groups at 3 months (greater in the platform group), there is a sharp increase in QoL of the platform group at six months, whereas those in the control group show a further drop in QoL. This is a probable illustration of the pattern of change in QoL that could be expected following increased use of ALADDIN, however in the absence of additional follow-up data at a later time point, it is difficult to comment on this. Similarly, since this significance was only found for a third of the sample that had rated their QoL on the EQ5D, this significant interaction should be interpreted with caution. Nonetheless, when we converted the QoL scores into percentages to make the outcome scores comparable across the three pilot sites, we found favorable results. These subsequent results showed that over the course of the pilot study, on average there was an improvement in the QoL of carers using ALADDIN, while there was a small deterioration in the QoL of the carers in the control group. Similarly feedback from the carers using ALADDIN suggested that they considered the system to have the potential to enhance their QoL.

In monitoring the PwD, the results showed a significant weight gain in patients in the platform group despite their significantly lower weight relative to control group at baseline. Older patients with dementia and particularly those with dementia as part of Parkinson's disease experience dysphagia (swallowing difficulties), have poor diets, and can experience weight loss $[33,34]$. Although presence of dysphagia was not formally assessed in this study, during the study period, the eating habits of the patients in the platform group were monitored through ALADDIN via the set questionnaires ('MY TASKS'), and referrals to dietary specialists were made when a problem was detected. This provides a possible explanation for the significant weight gain in the platform group. Hence frequent monitoring of patients was useful in providing key clinical data for improving the management of the PwD's care, including facilitating referrals and contact with other health professionals when necessary.

Although our results show statistically modest results, partly because of the small sample size, the relatively short duration of the pilot study, and the inconsistent assessment tools used for measuring QoL and depression in carers across the three pilot sites which restricted certain analyses; the results show promising trends that need to be further explored.

Since the use of ALADDIN was an active intervention, the inclusion of a sham treatment in the control group was difficult. It can be argued that in the absence of a placebo group the improvements observed in the platform group may reflect their experience of the placebo effect. Although this would threaten the validity of the benefits of ALADDIN as shown by the results, its relevance is limited. The purpose of ALADDIN is to alleviate feelings of burden and distress in carers of PwD. Thus the perceived or actual improvements observed in carers in the platform group relative to those in the control group illustrate that the use of ALADDIN does create a 'sense' of support which can be beneficial for the carers. The results also indicate that ALADDIN which brings together three distinct elements of support, information, and frequent monitoring, has the potential to reduce carer distress and burden and to improve QoL. Moreover our results highlight the importance of qualitative information in providing insight into some of the findings which may not reflect the true effects of an intervention due to time and resource constraints.

The application of telemedicine such as the ALADDIN platform can be easily implemented into existing personal computers and laptops, but requires further robust demonstration of its application and cost-efficacy in routine dementia care which remains a task for future studies. Future studies are encouraged to closely match the control and the experimental groups at baseline, as equivalence of groups at baseline may help to more clearly demonstrate the usefulness of a telemedicine system such as ALADDIN. Nonetheless, this study offers a randomized controlled evaluation of ALADDIN and establishes its merits.

ALADDIN is a platform that allows great flexibility and that can be tailored to individual cases and altered as changes in dementia dictate/necessitate over time. It offers convenient access from home, reducing the need to travel, which can be particularly useful for patients living in remote locations or those with physical restrictions. It also has the potential to reduce wider healthcare costs, such as the need for clinical resources and time to see patients, and can reduce waiting times and address needs quickly and more effectively. Thus, ALADDIN can be useful in not only supporting the carers of PwD and prolonging their care at home, but also in contributing to the better management of their care.

\section{ACKNOWLEDGMENTS}

This work was funded by a grant from the Ambient Assisted Living, European Commission. We are grateful to all the patients and their carers for their time and participation. 
Authors' disclosures available online (http://www.jalz.com/disclosures/view.php?id=2139).

\section{REFERENCES}

[1] Brookmeyer R, Johnson E, Ziegler-Graham K, Arrighi HM (2007) Forecasting the global burden of Alzheimer's disease. Alzheimers Dement 3, 186-191.

[2] (2007) Alzheimer's Society. Dementia UK, London.

[3] Wimo A, Winblad B, Jonsson L (2007) An estimate of the total worldwide societal costs of dementia in 2005. Alzheimers Dement 3, 81-91.

[4] Cohen D, Eisdorfer C (1988) Depression in family members caring for a relative with Alzheimer's disease. J Am Geriatr Soc 36, 885-889.

[5] Cohen D, Luchins D, Eisdorfer C, Paveza G, Ashford JW, Gorelick P, Hirschman R, Freels S, Levy P, Semla T, Shaw $\mathrm{H}$ (1990) Caring for relatives with Alzheimer's disease: The mental health risks to spouses, adult children, and other family caregivers. Behav Health Aging 1, 171-182.

[6] Eisdorfer C (1991) Caregiving: An emerging risk factor for emotional and physical pathology. Bull Menninger Clin 55, 238-247.

[7] Wolinsky FD, Callahan CM, Fitzgerald JF, Johnson RJ (1992) The risk of nursing home placement and subsequent death among older adults. J Gerontol 47, S173-182.

[8] Graham C, Ballard C, Sham P (1997) Carers' knowledge of dementia, their coping strategies and morbidity. Int J Geriatr Psychiatry 12, 931-936.

[9] Gaugler JE, Kane RL, Kane RA, Newcomer R (2005) Unmet care needs and key outcomes in dementia. J Am Geriatr Soc 53, 2098-2105

[10] Casper GR, Calvitti A, Brennan PF, Overholt JL (1995) ComputerLink: The impact of a computer network on Alzheimer's caregivers' decision-making confidence and skill. Medinfo 8(Pt 2), 1546.

[11] Mahoney DF, Tarlow BJ, Jones RN (2003) Effects of an automated telephone support system on caregiver burden and anxiety: Findings from the REACH for TLC intervention study. Gerontologist 43, 556-567.

[12] Finkel S, Czaja SJ, Schulz R, Martinovich Z, Harris C, Pezzuto D (2007) E-care: A telecommunications technology intervention for family caregivers of dementia patients. Am J Geriatr Psychiatry 15, 443-448.

[13] Glueckauf RL, Ketterson TU, Loomis JS, Dages P (2004) Online support and education for dementia caregivers: Overview, utilization, and initial program evaluation. Telemed $J$ E Health 10, 223-232.

[14] Marziali E, Donahue P (2006) Caring for others: Internet video-conferencing group intervention for family caregivers of older adults with neurodegenerative disease. Gerontologist 46, 398-403.

[15] Hayden LJ, Glynn SM, Hahn TJ, Randall F, Randolph E (2012) The use of Internet technology for psychoeducation and support with dementia caregivers. Psychol Serv 9, 215218.

[16] National Collaborating Centre for Mental H (2007) National Institute for Health and Clinical Excellence: Guidance.
In Dementia: A NICE-SCIE Guideline on Supporting People With Dementia and Their Carers in Health and Social Care, British Psychological Society, The British Psychological Society \& The Royal College of Psychiatrist., Leicester, UK.

[17] Williams K, Arthur A, Niedens M, Moushey L, Hutfles L (2013) In-home monitoring support for dementia caregivers: A feasibility study. Clin Nurs Res 22, 139-150.

[18] Mahoney FI, Barthel DW (1965) Functional evaluation: The Barthel Index. Md State Med J 14, 61-65.

[19] Folstein MF, Folstein SE, McHugh PR (1975) "Mini-mental state". A practical method for grading the cognitive state of patients for the clinician. J Psychiatr Res 12, 189-198.

[20] Zarit SH, Reever KE, Bach-Peterson J (1980) Relatives of the impaired elderly: Correlates of feelings of burden. Gerontologist 20, 649-655.

[21] Cummings JL, Mega M, Gray K, Rosenberg-Thompson S, Carusi DA, Gornbein J (1994) The Neuropsychiatric Inventory: Comprehensive assessment of psychopathology in dementia. Neurology 44, 2308-2314.

[22] Beck AT, Steer RA, Ball R, Ranieri W (1996) Comparison of Beck Depression Inventories -IA and -II in psychiatric outpatients. J Pers Assess 67, 588-597.

[23] Zung WW (1965) A self-rating depression scale. Arch Gen Psychiatry 12, 63-70.

[24] EuroQol Group (1990). EuroQol-a new facility for the measurement of health-related quality of life. Health Policy, 16, 199-208.

[25] Flanagan JC (1982) Measurement of quality of life: Current state of the art. Arch Phys Med Rehabil 63, 56-59.

[26] Jurica PJ, Leitten CL, Mattis S (2001) Dementia Rating Scale2: Professional manual.

[27] Roth M, Tomlinson BE, Blessed G (1967) The relationship between quantitative measures of dementia and of degenerative changes in the cerebral grey matter of elderly subjects. Proc R Soc Med 60, 254-260.

[28] Hely MA, Chey T, Wilson A, Williamson PM, O'Sullivan DJ, Rail D, Morris JG (1993) Reliability of the Columbia scale for assessing signs of Parkinson's disease. Mov Disord 8, 466-472.

[29] Teri L, Truax P, Logsdon R, Uomoto J, Zarit S, Vitaliano PP (1992) Assessment of behavioral problems in dementia: The revised memory and behavior problems checklist. Psychol Aging 7, 622-631.

[30] Yesavage JA (1988) Geriatric depression scale. Psychopharmacol Bull 24, 709-711.

[31] Lawton MP, Brody EM (1969) Assessment of older people: Self-maintaining and instrumental activities of daily living. Gerontologist 9, 179-186.

[32] Charlson ME, Pompei P, Ales KL, MacKenzie CR (1987) A new method of classifying prognostic comorbidity in longitudinal studies: Development and validation. J Chronic Dis 40, 373-383.

[33] LeClerc CM, Wells DL, Sidani S, Dawson P, Fay J (2004) A feeding abilities assessment for persons with dementia. Alzheimers Care Q 5, 123-133.

[34] Johnston BT, Li Q, Castell JA, Castell DO (1995) Swallowing and esophageal function in Parkinson's disease. Am J Gastroenterol 90, 1741-1746. 